\title{
Clinical Use of Extracorporeal Shockwave Therapy (ESWT) for the Treatment of Carpus Valgus Deformities in Young Foals: A Retrospective Study of 64 Cases (2006-2009)*
}

\author{
Christian Bussy", Francois Auzas, Juan A. Muñoz \\ Clinique Vétérinaire du Grand Renaud, Le Grand Renaud, Saint Saturnin, France \\ Email: "contact@vetoequin.fr
}

Received November 15, 2012; revised December 17, 2012; accepted January 18, 2013

\begin{abstract}
The purpose of this study was to report the use and assess the effects of extracorporeal shockwave therapy (ESWT) for the treatment of carpal joint valgus deformities (CJVDs) in young foals. Only foals with CJVDs greater than $5^{\circ}$ were included in the study. Foals were assigned to 3 treatment groups based on their degree of CJVD measured during the initial evaluation: valgus deformity (VD) of $5^{\circ}$ to $8.9^{\circ}$ (Group 1), VD of $9^{\circ}$ to $11.9^{\circ}$ (Group 2) and VD greater than or equal to $12^{\circ}$ (Group 3). ESWT was applied on the convex side of the angular deformity immediately following the initial radiographic evaluation. Foals were subsequently evaluated clinically and radiologically followed by treatment every 10 days until resolution of the VD, with resolution defined as a deviation less than 5 degrees. Each treatment group received specific exercise, hoof trimming and hoof/shoe extension recommendations. Sixty-four (64) foals were included in the study; ages ranged from 8 to 60 days old at inclusion in the study with a mean age of 26.7 days. Of the 28 foals included in Group 1, 10 had bilateral CJVD. There were 21 in Group 2, and 15 in Group 3. Treatment success was defined as a VD angle less than $5^{\circ}$, and was reached in all foals in Groups 1 and 2. Five (5) foals in Group 3 completed the study with a VD angle of $5^{\circ}$ to $6.7^{\circ}$ at the last radiographic assessment. No major complications were observed during the study. In conclusion, ESWT in conjunction with controlled exercise, hoof trimming and hoof/shoe extensions corrected severe CJVDs in young foals. The use of ESWT eliminates possible negative side effects of general anesthesia and surgical techniques to treat VDs. Future studies should include a control population, more severe cases, other types of angular limb deformities, and older foals.
\end{abstract}

Keywords: Angular Limb Deformity; Foal; Growth Plate; Extracorporeal Shockwave Therapy Valgus; Carpus

\section{Introduction}

Angular limb deformities (ALDs) are the most commonly reported abnormalities diagnosed in foals and most frequently affect the carpal joint $[1,2]$. Further, ALDs can be the primary reason for euthanasia in foals over 2 weeks of age [3]. Although corrective trimming and shoeing, as well as controlled exercise, can in many cases successfully correct ALDs, a significant number of foals are treated surgically. Surgical treatment is usually associated with higher risks and costs as well as potential permanent cosmetic changes at the surgery site.

Growth at the level of the metaphyseal region of the physis represents both the majority of the longitudinal growth in the long bone, and a feedback loop regulated via the periosteum/perichondrium which is centered on

\footnotetext{
${ }^{*}$ Conflict of interest statement: None of the authors has any financial or personal relationships that could inappropriately influence or bias the content of the paper.

${ }^{\#}$ Corresponding author.
}

the following molecules: parathyroid hormone-related protein $(\mathrm{PTHrP}), \mathrm{PTH} / \mathrm{PTHrP}$ receptors $(\mathrm{PTHrPr})$ and $\mathrm{In}-$ dian Hedgehog (Ihh) [4].

Several surgical techniques aimed to locally accelerate or decelerate growth plate activity have been described [1]. Selection of a surgical technique depends on the age of the foal, the degree of angular limb deformity, the anatomical site, and whether the deformity is a varus or a valgus. The use of extracorporeal shockwaves has been suggested as an alternative to these surgical techniques for the treatment of ALDs in foals $[5,6]$.

Shockwaves are sound waves characterized by a very fast and steep rise in pressure followed by a negative pressure period. They are defined as transient pressure oscillations that propagate in three dimensions [7]. The mechanisms by which shock waves affect bone and growth plates are not fully understood. It is believed that shock waves can stimulate neovascularization and influence numerous biological factors as FGF-2 and TGF- $\beta$ of injured tissues $[8,9]$. Experimental work in neonatal rats 
has suggested that extracorporeal shockwave therapy (ESWT) may induce focal growth plate dysplasia [10]. The use of ESWT over the growth plate in 3 experimental ponies showed a growth retardation effect on the treated side of the physis (Boening unpublished data). Positive effects using ESWT were also seen in the treatment of ALDs in a prospective clinical trial in foals [5].

The objective of this study was to radiographically evaluate the effect of ESWT on carpal joint VD. We hypothesised that ESWT is an effective, non-invasive method for the treatment of these types of VDs even in severe cases.

\section{Materials and Methods}

\subsection{Animals}

Foals included in the study were evaluated and treated for CJVD at the Grand Renaud Veterinary Clinic between January 2006 and September 2009.

\subsection{Inclusion and Exclusion Criteria}

Foals with a CJVD of at least $5^{\circ}$ were included in the study. Foals with carpal joint laxity, rotational deformities, incomplete ossification of the cuboid bones or other abnormalities such as foot abscesses, traumatic injuries or bone fractures were excluded.

\subsection{Study Design}

Animals were assigned to 3 treatment groups based on their degree of CJVD measured during the initial evaluation.

\begin{tabular}{ccc}
\hline Group 1 & Group 2 & Group 3 \\
\hline $5^{\circ}$ to $8.9^{\circ}$ & $9^{\circ}$ to $11.9^{\circ}$ & $12^{\circ}$ to $21.6^{\circ}$ \\
\hline
\end{tabular}

\subsection{Evaluation Protocol}

The VD diagnosis was made using a combination of clinical and radiographic techniques. On presentation, foals were evaluated in a standing position as well as walking on a straight line. Presence of joint laxity and uneven wear of the hoof wall were documented. Radiographs were obtained using a Philips Eleva computer system and used to measure the degree of limb deformity.

Foals were sedated for radiography using a combination of romifidine (Sedivet, Boehringer Ingelheim) 0.04 $\mathrm{mg} / \mathrm{kg}$ bwt (i.v.), and butorphanol (Dolorex, Intervet) $0.01 \mathrm{mg} / \mathrm{kg}$ bwt (i.v.). The limb was carefully positioned to ensure that sedation did not interfere with the measurement of the VD angle. Radiographs were obtained using $38 \times 45 \mathrm{~cm}$ cassettes. In all cases dorsopalmar (DP) views of the carpal joint(s) were obtained. Radiographs were centered on the carpus including a significant portion of the radius and metacarpus with the second and fourth metacarpal bones being as parallel as possible. The degree of VD was measured as the angle between the intersection of the line that longitudinally bisects the diaphysis of the metacarpus, and the line that longitudinally bisects the diaphysis of the radius (Figure 1). When necessary, additional views were taken to rule out other problems. All radiographs and measurements were performed or supervised by the same clinician.

\subsection{ESWT Procedure}

Foals were treated by ESWT immediately after radiographs and measurements were taken. ESWT was applied on the medial aspect of the distal diaphysis and metaphysis of the radius (Figure 2). The area to be treated

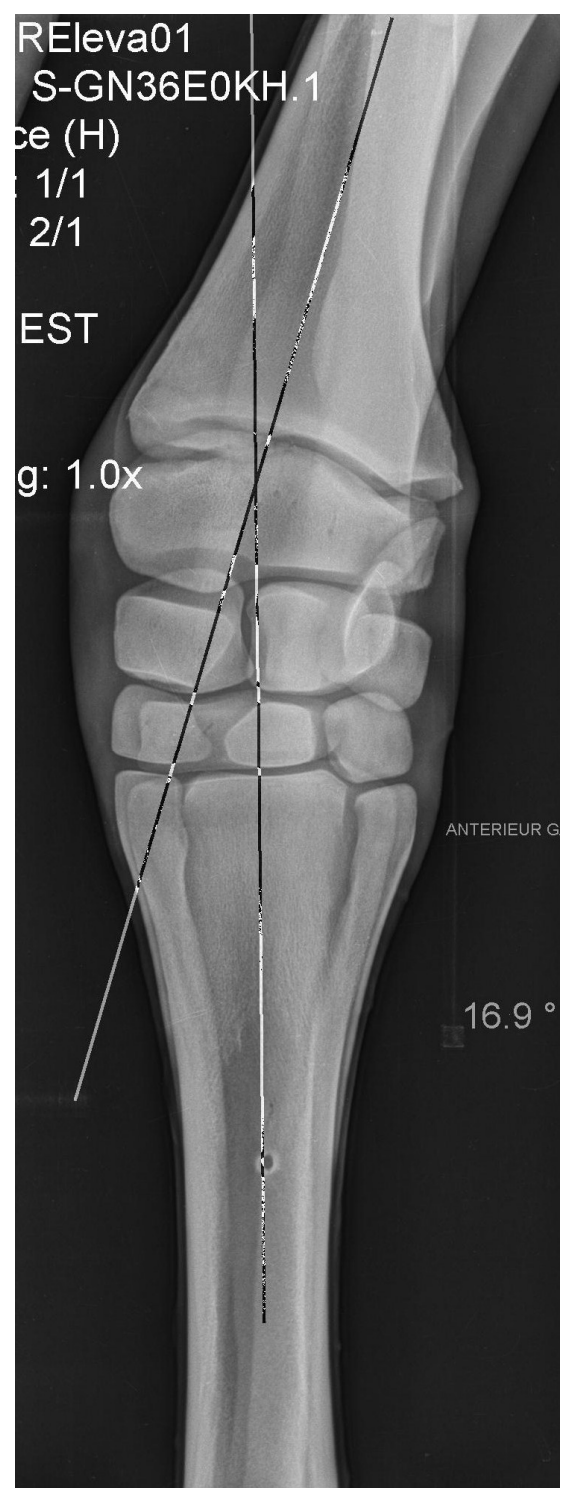

Figure 1. Dorsopalmar radiography of a 40 days old, group 3 foal showing CJVD at first treatment. The VD angle (16.9 degrees), performed by the insertion of both lines, is shown. 


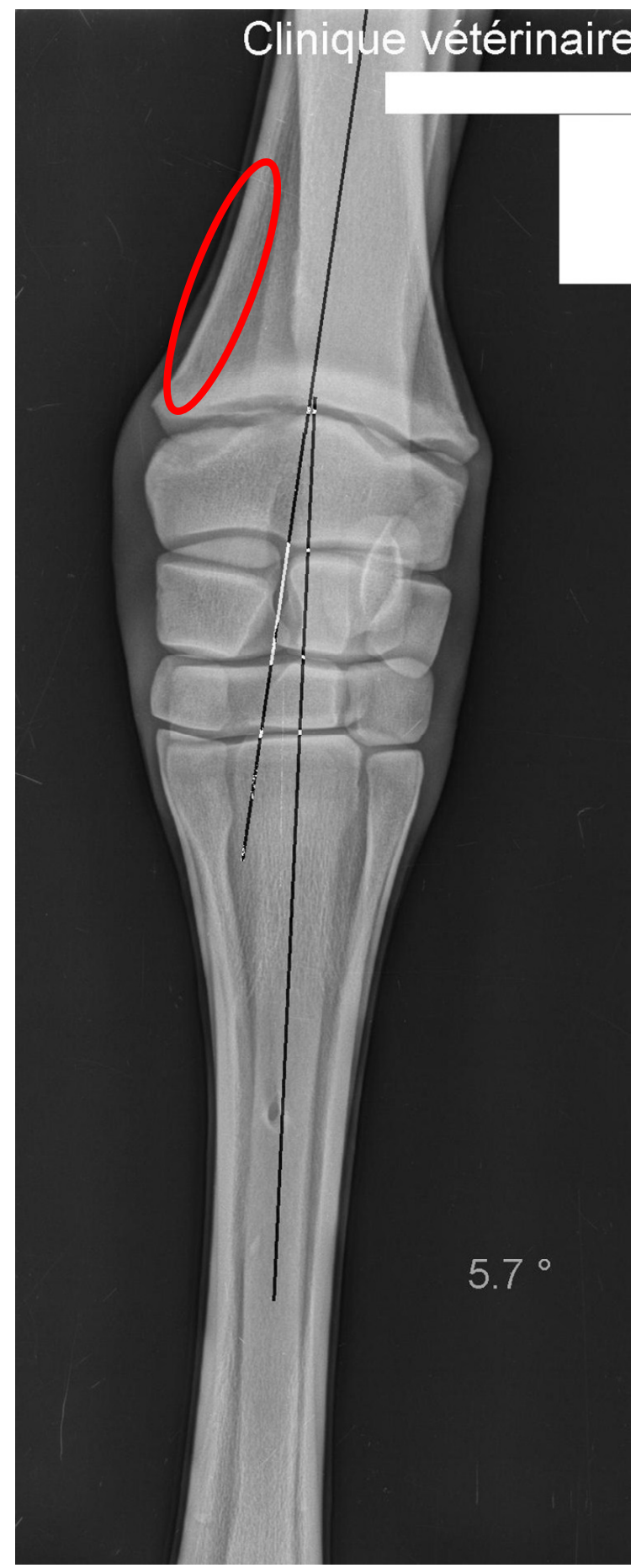

Figure 2. Dorsopalmar radiography of the foal represented in figure 1 twenty days after the first treatment. A reduction in VD angle to 5.7 degrees is observed. The area of ESWT application is shown (red ellipse).

was clipped and ultrasound coupling gel applied. Two thousand impulses generated by a ballistic shockwave source (Masterpuls MP100-Störz medical-TägerwilenSuisse) were applied at a frequency of $15 \mathrm{~Hz}$, under 2.5 bars of pressure using a $15 \mathrm{~mm}$ applicator. The time re- quired to perform the procedure was recorded in each case.

Following the first treatment with ESWT, foals were re-evaluated clinically and radiographically as well as treated every 10 days, until resolution of the ALD. Exercise recommendations during the course of therapy were based on the degree of ALD. Foals in Groups 1 and 2 were stabled with their dams and had access to a small paddock to allow for a controlled exercise schedule. Foals in Group 3 were on stall rest until the ALD reached less than $12^{\circ}$. Occasionally, orthopaedic treatments such as hoof trimming, hoof/shoe extensions and glue-on shoes were performed and re-evaluated every 20 days until resolution of the VD. Follow-up phone calls were made to the owners or referring veterinarians between 1 and 3 months after the last visit.

\section{Results}

Seventy-eight (78) foals were evaluated for CJVD. Sixtyfour (64) met the inclusion criteria and were included in the study. Fourteen (14) were excluded from the study due to various reasons including joint laxity ( 2 foals), rotational deformities ( 2 foals), incomplete ossification of the cuboid bones (2 foals), foot abscess ( 2 foals), and traumatic injury or fracture of the affected limb (6 foals).

Foals included in the study were 8 to 60 days old (mean $=26.7$ days, $\mathrm{SD}=9.4$ days). Breeds included were Thoroughbred $(n=20)$, French Standardbred $(n=20)$, French Warmblood $(n=7)$, French Pony $(n=2)$, French Saddlebred $(n=2)$, Arabian $(n=2)$, Dutch Warmblood $(\mathrm{n}=2)$, and unknown $(\mathrm{n}=9)$. Of the 64 foals included in the study, a total of 74 CJVDs were diagnosed (10 foals suffered from bilateral CJVD).

Twenty-eight foals were included in Group 1, of which 10 had bilateral CJVD. There were 21 foals in Group 2 and 15 in Group 3. Thirty-three foals (12 from Group 1, 13 from Group 2 and 8 from Group 3) had been unsuccessfully treated conservatively (hoof trimming and stall rest) prior to referral to this clinic. In this study, hoof trimming was performed on 13 foals in Group 1 and 10 foals in Group 2; medial acrylic hoof extensions were applied on 11 foals in Group 2; and glue-on shoes with medial extension were applied to all foals in Group 3. No foals had further orthopaedic treatment or ESWT after their last visit to the clinic.

Treatment success, defined as a VD angle was less than $5^{\circ}$, was reached in all foals after an average of 1.5 (1 to 3 ) and 2.2 (1 to 4) shockwave treatments in Groups 1 and 2 respectively. Foals in Group 3 required an average of 2.5 (2 to 6) shockwave treatments. Five foals in Group 3 completed the study with a VD angle of $5^{\circ}$ to $6.7^{\circ}$ at the last radiographic assessment. Follow-up information on all foals was obtained by phone 1 to 3 months following the last evaluation at the clinic and showed that all 
foals in all treatment groups reached a satisfactory limb conformation according to the owner or referring veterinarian.

The fastest response to ESWT was demonstrated in a foal (Group 3) with an initial VD of $21^{\circ}$ that showed an improvement of $11^{\circ} 10$ days after the first treatment.

ESWT was well tolerated by all foals that were sedated only once prior to the radiography. Mean duration of treatment was $20 \mathrm{~min}$, which included $10 \mathrm{~min}$ for radiography and $10 \mathrm{~min}$ for ESWT. Three foals presented with a 2 out of 5 degree lameness (AAEP grading system) the day after the first ESWT. Causes for the lameness, such as localized swelling, heat or any other abnormality at the treatment site were not reported by owners or referring veterinarians. In all cases, lameness resolved within 24 hours without treatment.

\section{Discussion}

Over a 3-year period (from 2006 to 2009) only 4 foals with CJVD were treated surgically at the Grand Renaud Veterinary Clinic due to owner request. All other foals were treated by ESWT. Prior to implementing the use of ESWT, 15 to 20 foals presenting with VDs were treated surgically at the clinic every year.

The use of ESWT was shown to be effective for the treatment of VD in this study with resolution of all VDs in Groups 1 and 2. In Group 3, only 5 foals continued to exhibit a VD greater than $5^{\circ}$ at the evaluation at the clinic. The owners of these 5 foals were given the option to continue ESWT but opted not to as they were satisfied with the outcome to date. Follow-up phone calls 1 to 3 months later confirmed that the foals were doing well and that the residual VDs were acceptable to the owners. It is important to note that when not severe, carpal VD may be accepted by Thoroughbred owners who recognize that in this breed, carpal VD is correlated to a protective mechanism that results in a decrease in the odds of carpal fracture and carpal effusion as the carpal angle increases [11].

Resolution of the VD was achieved after a mean of 2.5 treatments applied according to Bathe's protocol [5]. In this study, according to the manufacturer's data, the shock wave generator provides an energy flux density of $0.38 \mathrm{mj} / \mathrm{mm}^{2}$. The use of a mild dose of energy in experimental protocols using a rat model does not appear to damage joint cartilage structure but does impact chondrocyte viability and membrane permeability [12-14]. Moreover, ESWT seems to promote cell differentiation toward bone cell via P38 kinase mediation [15]. This impact in cell viability, membrane permeability and cell differentiation towards bone cells, in addition to the growth retardation on the physis reported by Yeaman et al. [10], could create growth retardation at the level of the growth plate which would explain the study results of this study.

Several adverse effects related to ESWT have been reported in humans and animals at the energy level used in this study. These effects include excoriation of the skin at the site of the hand piece application and the development of small circular areas of alopecia with subsequent development of white hair [16]. The only adverse effect in this study was observed in 3 foals that presented a 2 out of 5 degree lameness the day after the first treatment with ESWT (lameness resolved within 24 hours without treatment). Although pain at the treatment site was likely to be the cause of lameness, clinical signs such as localized swelling or heat were not observed. None of the negative effects observed in other experimental studies such as petechia, hematoma, and skin necrosis at the level of the treated area were observed in this study. While it is possible for overcorrection to occur following transphyseal bridging (surgical growth retardation procedure) this was not observed in any study animals.

It is likely that some foals presented in this study with mild deformities would have improved without ESWT. However, 34 of the foals included in this study were referred to the clinic because of prior failure or deterioration of their VD (increase in angle deviation) despite classic conservative treatments such as hoof trimming and stall rest. As reported by Bathe et al. [5] the clinical impression is that foals with mild deformities treated with hoof trimming or hoof/shoe extensions need greater time for ALD resolution, and the degree of correction is less significant than obtained in foals treated with ESWT. These conclusions are subjective and a control group should be used to obtain objective results. Further the mean foal age is similar with the Bathe study [5]. The young age of some foals included in this study can be explained by the need for correcting VDs as early as possible as these foals will likely be sold before they are yearlings. Foals with severe carpal deformities (deviation greater than $15^{\circ}$ or greater than $10^{\circ}$ after 4 weeks of age) normally need surgical treatment to achieve ALD resolution [17]. In the present study most foals with severe deformities were corrected using ESWT, hoof extensions, and control exercise. Future studies should include negative and positive (surgical) control groups, which may be difficult to achieve in the context of a private practice where clients may want to impose a preference of one treatment over another.

All foals were treated during high growth plate activity (less than 4 months at the distal radius physis). This study does not show the effect of ESWT on VDs during slow growth plate activity. It is likely that ESWT in older foals may be less effective than in young foals. This study did not include varus deformities due to insufficient case load. 
For some foals, a linear correction in the degree of deviation was not observed after each administration of ESWT. We hypothesize that a linear growth is not present at the level of the growth plate, which may explain why some foals may only need 1 treatment while others need 2 or 3 to achieve the same degree of correction. These observations support studies showing the nonlinear growth of foal from birth to weaning [18].

Two methods exist to evaluate VDs radiographically. In addition to the method described in this study, a second method consists of drawing parallel lines to the growth plate and radiocarpal, intercarpal and carpometacarpal articular surface. The intersection of these lines defines the deviation and the pivot point. This second technique is not as accurate as the first method but allows multifactorial deviations to be evaluated. These two methods cannot fully assess deformities in two planes (frontal and sagittal for example). If deformities in two planes are suspected, two radiographs (DP and lateromedial views) and a normogram can determine the true angle of the deformity, which routinely exceeds the angle measured in one plane and its orientation [19]. Given that the method chosen to measure VD angles in this study could result in significant variability, all measurements were performed and/or verified by the same person. Most foals included in this study had to be sedated to be handled and radiographs to be taken. In order to minimize the potential effect of sedation on the VD angle measurements, foals were manually squared.

The advantages associated with the use of ESWT are that this 20 minute procedure is non-invasive, can be performed under light sedation on an outpatient basis or in the field, has no risk of infection, and does not result in any noticeable cosmetic changes of the treated area in equine subjects.

\section{Conclusion}

In conclusion, we showed that ESWT along with controlled exercise, hoof trimming and hoof/shoe extensions successfully treats CJVDs even in severe cases. In future studies, a control group should be used. More severe cases, older foals and those affected with varus deformities should also be included.

\section{REFERENCES}

[1] J. A. Auer, "Angular Limb Deformities," In: J. A. Auer and J. A. Stick, Eds., Equine Surgery, 3rd Edition, Saunders Elvesier, St Louis, 2006, pp. 1138-1142.

[2] D. O'Donohue, F. Smith and K. Strickland, "The Incidence of Abnormal Limb Development in the Irish Thoroughbred from Birth to 18 Months," Equine Veterinary Journal, Vol. 24, No. 4, 1992, pp. 305-309. doi:10.1111/j.2042-3306.1992.tb02841.x

[3] P. Morley and H. Towsend, "A Survey of Reproductive
Performance in Thoroughbred Mares and Mobidity, Mortality and Athletic Potential of Their Foals," Equine Veterinary Journal, Vol. 29, No. 4, 1997, pp. 290-297. doi:10.1111/j.2042-3306.1997.tb03126.x

[4] A. Vortkamp, K. Lee and B. Lanske, "Regulation of Rate of Cartilage Differentiation by Indian Hedgehog and PTH-Related Protein," Science, Vol. 273, No. 5275, 1996, pp. 273-613.

[5] A. P. Bathe, D. S. Rowlands and K. J. Boening, "Treatment of Angular Limb Deformities Using Radial Extracorporeal Therapy: A Prospective Clinical Trial," Proceedings of the 9th International Congress of World Equine Veterinary Association, Marrakech, 22-26 January 2006, pp. 167-168.

[6] C. Bussy, "Deviations Angulaires et Ondes de Choc," Le Point Vétérinaire, Vol. 275, 2007, pp. 39-43.

[7] F. Ueberle, "Extracorporeal Shockwaves in Orthopaedics," In: W. Siebert and M. Buch, Eds., Shockwave Technology, Springer, Berlin, 1997, pp. 59-87.

[8] D. Bolt, D. Burba and J. Hubert, "Determination of Functional Changes in Palmar Nerves after Nonfocused ESWT in Horses," American Journal of Veterinary Research, Vol. 65, No. 12, 2004, pp. 1714-1718. doi:10.2460/ajvr.2004.65.1714

[9] J. Hausdorf, B. Sievers, M. Schmitt-Sody, V. Jansson, M. Maier and S. Mayer-Wagner, "Stimulation of Bone Growth Factor Synthesis in Human Osteoblasts and Fibroblasts after Extracorporeal Shock Wave Application," Archive of Orthopaedic Traumatology Surgery, Vol. 24, No. 1, 2010, pp. 10-18.

[10] L. Yeaman, C. Jerome and D. Mccullough, "Effects of Shock Waves on the Structure and Growth of the Inmature Rats Epiphysis," Journal of Urology, Vol. 141, 1989, pp. 670-674.

[11] T. Anderson and C. McIlwraith, "Longitudinal Development of Equine Conformation from Weanling to Age 3 Years in the Thoroughbred," Equine Veterinary Journal, Vol. 36, No. 7, 2004, pp. 536-538.

[12] C. Byron, A. Stewart, B. Benson, B. Tennent-Brown and J. Foreman, "Effects of Radial Extracorporeal Shock Wave Therapy on Radiographic and Scintigraphic Outcomes in Horses with Palmar Heel Pain," Veterinary and Comparative Orthopaedics and Traumatology, Vol. 22, No. 2, 2009, pp. 113-118.

[13] C. Lischer, S. Ringer, M. Schnewlin, I. Imoboden, A. Furst, M. Stockli and J. Auer, "Treatment of Chronic Proximal Suspensory Desmitis in Horses Using Focused Electrohydraulic Shockwave Therapy," Schweiz Arch Tierheilkd, Vol. 148, No. 10, 2006, pp. 561-568. doi:10.1024/0036-7281.148.10.561

[14] M. Loew, W. Daeke, D. Kusnierczak, M. Rahmanzadeh and V. Ewerbeck, "Shock-Wave Therapy Is Effective for Chronic Calcifying Tendinitis of the Shoulder," Journal of Bone Joint Surgery British, Vol. 81, No. 5, 1999, pp. 863-867.

[15] Y. J. Chen, F. S. Wang, C. J. Wang, D. Y. Kuender, Y. R. Kuo and H. C. Huang, "Shockwave Attenuates InterleukinIbeta, Cydooxygenase-2 and Prostaglandin E2 Receptor Expressions during Healing of Collagenase-Induced Achil- 
les Tendonitis in Rats," Proceedings of a Conference on the 7th Congress of the ISMT, Kaohsiung, 1-4 April 2004, pp. 116-119.

[16] O. Crowe, "Treatment of 45 Cases of Chronic Hindlimb Proximal Suspensory Desmitis by Radial," Proceeding of the American Association of Equine Practitioners, Orlando, 4-8 December 2002, pp. 322-325.

[17] J. Auer, R. Martens and E. Williams, "Periosteal Transection for Correction of Angular Limb Deformities in Foals," Journal of American Veterinary Medical Association, Vol.
181, No. 5, 1982, pp. 181-459.

[18] P. Morel, A. Bokor, C. Rogers and E. Firth, "Growth Curves from Birth to Weaning for Thoroughbred Foals Raised on Pasture," New Zealand Veterinary Journal, Vol. 55, No. 6, 2007, pp. 319-325. doi: $10.1080 / 00480169.2007 .36788$

[19] H. Bar and H. Breitfuss, "Analysis of Angular Deformities on Radiographs," Journal of Bone \& Joint Surgery British, Vol. 71, No. 4, 1989, pp. 710-711. 